\title{
Virtuelle Gremiensitzungen: Sinnvolle Ergänzung nicht nur in Krisenzeiten
}

\author{
Extrakt des Beitrags "Virtuelle Gremiensitzungen im Rahmen einer \\ nachhaltigen Unternehmensführung" von Thomas Kremer, Luka \\ Mucic, Ralf P. Thomas und Axel v. Werder* erschienen in: Der \\ Betrieb, 74. Jg. 2021, S. 1145-1158.
}

Im Zuge der pandemiebedingten Lockdowns haben sich die Modalitäten der Vorstands- und Aufsichtsratssitzungen seit dem letzten Jahr durch den weitgehenden Übergang auf virtuelle Formate grundlegend geändert. Die Mitglieder des Roundtable des Berlin Center of Corporate Governance (BCCG) kommen auf der Grundlage der bisherigen Erfahrungen zu dem Ergebnis, dass sich virtuelle Gremiensitzungen als Instrument der Corporate Governance in der Corona-Krise durchaus bewährt haben. Videokonferenzen werden Präsenzsitzungen in normalen Zeiten nicht vollständig ersetzen, jedoch sinnvoll ergänzen können. Beide Sitzungsformate haben jeweils spezifische Stärken und Schwächen.

\section{Bewertung virtueller Gremiensitzungen}

Nach Einschätzung der Mitglieder des Roundtable des BCCG hat ein Großteil aller Vorstands- und Aufsichtsratssitzungen während der bisherigen Phasen des Lockdowns als Videokonferenz stattgefunden. Die weitgehende Abkehr von Präsenzsitzungen war aus der Not geboren. Die virtuellen Formate erlaubten Vorstand und Aufsichtsrat trotz weitreichender Reise- und Kontaktbeschränkungen ihre Unternehmen zu leiten und zu überwachen. Auf mittlere und längere Sicht stellt sich die Frage, ob und inwieweit eine Digitalisierung der Gremiensitzungen als Element guter Corporate Governance systematisch etabliert werden soll.

\footnotetext{
Dr. Thomas Kremer | ehemaliges Mitglied des Vorstands | Telekom AG Luka Mucic | Mitglied des Vorstands I SAP SE

Prof. Dr. Ralf P. Thomas | Mitglied des Vorstands | Siemens AG

Prof. Dr. Axel v. Werder | Lehrstuhl für Betriebswirtschaftslehre, insbesondere Organisation und Unternehmensführung | Technische Universität Berlin
} 
Aufschlussreich ist etwa mit Blick auf die Überwachungseffizienz des Aufsichtsrats eine Gegenüberstellung von reinen Videokonferenzen, hybriden Formaten und Präsenzsitzungen hinsichtlich der Kriterien Qualität, Aufwand und Sicherheit der Überwachung. Hierbei gilt es nicht nur den regulatorischen Rahmen, sondern neben sach- insbesondere auch verhaltensbezogene Faktoren zu berücksichtigen. Stichworte sind in diesem Zusammenhang beispielsweise Diskussionskultur, Gruppendynamik, "Onboarding" oder "Socializing".

Die Mitglieder des BCCG-Roundtable fassen ihre Erfahrungen mit virtuellen Sitzungen des Aufsichtsrats in zehn Thesen zusammen:

These 1: Rechtliche Vorschriften stehen virtuellen Sitzungen des Aufsichtsrats prinzipiell nicht entgegen.

These 2: Virtuelle Sitzungen können ohne aktive Sitzungsleitung den Detailierungsgrad der Diskussionen mindern, erhöhen aber auch die Reaktionsgeschwindigkeit des Aufsichtsrats.

These 3: Die Gruppendynamik von physischen Zusammenkünften lässt sich bei virtuellen Sitzungen kaum vollständig nachbilden.

These 4: Der Aufwand virtueller Sitzungen ist - von den Kosten für die technische Infrastruktur abgesehen - merklich geringer als der von Präsenzsitzungen.

These 5: Die Gewährleistung von Sicherheit und Vertraulichkeit ist eine besondere Herausforderung virtueller Sitzungen.

These 6: Unter Abwägungen von Qualität und Aufwand der Überwachung empfehlen sich im Normalfall Videokonferenzen für Routinetätigkeiten des Aufsichtsrats und Präsenzsitzungen für Aufgaben großer Komplexität, Tragweite sowie Sensibilität.

These 7: Es kann als Element guter Corporate Governance gelten, virtuelle Aufsichtsratssitzungen im jährlichen Überwachungszyklus nicht nur ausnahmsweise vorzusehen.

These 8: Videokonferenzen stellen formatspezifische Anforderungen an die Vorbereitung und Durchführung der Sitzungen.

These 9: Der Aufsichtsratsvorsitzende ist bei virtuellen Sitzungen in besonderem Maße gefordert.

These 10: Für den Vorstand bilden (teil-)virtuelle Sitzungen als regelmäßige Ergänzungen physischer Zusammenkünfte bereits seit Längerem bewährte Praxis.

\section{Empfehlungen zur Einführung und Gestaltung virtueller Sitzungsformate}

In Form einer Checkliste stellen die Mitglieder des BCCG Punkte zusammen, die bei der Planung und Leitung virtueller Aufsichtsratssitzungen bedacht werden sollten: 
Checkliste

Nützliche Vorfeldüberlegungen zur Einführung virtueller Sitzungsformen

I. Allgemein zu klärende Fragen vor der Nutzung virtueller Sitzungselemente

Ist noch ausreichend Zeit für eine Sitzungsplanung einschließlich der Klärung der

Organisation und Technik der Sitzungsformate?

Empfehlung: regelmäßige frühzeitige Sitzungsplanung über Ein-oder Zweijahreszeitraum

Empfehlung: Termine unabhängig vom Sitzungsformat mit hinreichendem Vorlauf vor der eigentlichen Einladung mit "Save-the-Date"reservieren

Ist noch ausreichend Zeit für eine Sitzungsplanung einschließlich der Klärung der Organisation und Technik der Sitzungsformate?

Welche Sitzungen sollen virtuell oder als Präsenzveranstaltung durchgeführt werden?

Empfehlung: gewisse Mindestanzahl von Präsenzveranstaltungen (etwa Hälfte) sollte nicht unterschritten werden (sofern keine Ausnahmesituation vorliegt)

Welche Themen sind bei welchen Sitzungen zu erwarten und eignen sich diese gut für virtuelle Sitzungen?

Empfehlung: komplexe Themen, die eine intensive (bzw. kritische) Diskussion mit einer Vielzahl von Wortbeiträgen erwarten lassen (z.B. Strategie-oder Haftungsdurchsprachen oder Diskussionen von M\&A-Projekten), tendenziell eher für eine Präsenzsitzung geeignet

Empfehlung: Bilanzsitzung des Aufsichtsrats und die Jahresabschlusssitzung des Prüfungsausschusses sollten in der Regel bzw. wenn möglich als Präsenzsitzungen abgehalten werden

Soll im Aufsichtsrat eine Möglichkeit gegeben werden, vorab über Fragen des Sitzungsformats zu diskutieren?

Empfehlung: ggf. vor der Einführung virtueller Sitzungen eine grundsätzliche Diskussion - bspw. über Festlegung einer Mindestzahl von Präsenzsitzungen pro Jahr oder zusätzlichen Trainingsbedarf für die Durchführung virtueller Sitzungsformen - führen

Sollen auch hybride Sitzungsformen vorgesehen werden?

Empfehlung: Hybridformen nur sparsam, mit Bedacht und nur im Einzelfall einsetzen, da etwaigen Vorteilen auch gewisse Nachteile (etwa erhöhter bzw. doppelter Aufwand) gegenüberstehen

Soll auch bei der Planung von Präsenzsitzungen parallel die Infrastruktur für eine virtuelle oder hybride Sitzung verfügbar sein?

Empfehlung: zumindest bei größeren, internationalen Aufsichtsräten: virtuelle Lösung als back-up

Wird neben der virtuellen Sitzung eine virtuelle Unterlagenführung beabsichtigt?

Empfehlung: Entscheidung zur Einrichtung eines virtuellen Boardrooms muss sehr gut vorbereitet werden und mit langem Vorlauf erfolgen 
II. Bei Entscheidung zugunsten virtueller Sitzungsformate zusätzlich zu klären:

Ist der Aufsichtsratsvorsitzende hinreichend auf seine besondere Moderatorenrolle bei virtuellen Sitzungen vorbereitet?

Empfehlung: eventuell spezifische Trainings für den Aufsichtsratsvorsitzenden sinnvoll

Empfehlung: Hinweise bzw. Erläuterungen zu Ablauf und Modalitäten zu Beginn der Sitzung (etwa welche Verhaltensregeln sind zu beachten, wie erfolgen Wortmeldungen bzw. mit welcher Funktion der Konferenz-Software kann man sich zu Wort melden)

Empfehlung: Sitzungsleiter sollte sich um eine besonders aktive Leitung der Diskussion (gezieltes Nachhaken, Einbindung weiterer Aufsichtsratsmitglieder) bemühen

Empfehlung: Wortmeldungen mittels elektronischer Chat-Funktionen anzeigen lassen

Empfehlung: Unterstützung des Sitzungsleiters durch eine weitere Person (Protokollführer), die virtuellen Chat kontinuierlich überwachen und Rednerliste führen kann

Was passiert in Notfallszenarien (Netzstabilität, technische Probleme)?

Empfehlung: Möglichkeit einer Telefoneinwahl mit einer geordneten Funktion der Wortmeldung bzw. -erteilung als back-up; bereits bei der Einladung mit zu kommunizieren

Empfehlung: "Notfall-Tel.-Nummer"kommunizieren, die den Teilnehmern bei technischen Problemen zur Verfügung steht (der so zu erreichende Ansprechpartner steht hierfür selbst zur Verfügung und/oder koordiniert die erforderliche Hilfe)

Sollen mobile Endgeräte wie Laptops oder Tablets ausgegeben oder private Endgeräte als Hardware genutzt werden?

Empfehlung: seitens des Unternehmens in jedem Fall adäquate Unterstützung der Aufsichtsratsmitglieder anbieten

Empfehlung: bei Ausgabe von Endgeräten Managed Security einsetzen

Empfehlung: bei Nutzung privater Endgeräte muss Aufsichtsratsmitgliedern bewusst sein, dass sie selbst für Beachtung der erforderlichen Sicherheitsstandards verantwortlich sind

Hat sich das Unternehmen (IT-Betreuerstab) mit der Auswahl einer geeigneten

Videokonferenz-Software oder sonstiger Lösungen auseinandergesetzt?

Empfehlung: technische Infrastruktur muss geeignet sein, die friktionslose Teilnahmemöglichkeit aller Mitglieder und Gäste verlässlich anzubieten

Empfehlung: Erwerb einer Lizenz zur Nutzung der Videokonferenz-Software erst nach eingehendem Auswahlprozess, um technisch nahtlose Anbindung der eingesetzten Videokonferenz-Software an den digitalen Sitzungsraum zu ermöglichen

Empfehlung: Nutzung sonstiger technischer Möglichkeiten der VideokonferenzSoftware (bspw. einer "Raise hands"-Möglichkeit, Chat-Funktion)

Ist eine (Simultan-)Übersetzung erforderlich?

Empfehlung: etwaig erforderliche Übersetzer rechtzeitig vor den Sitzungsterminen reservieren (in der Regel wechseln sich die Übersetzer nach einiger Zeit ab bzw. sind mehrere erforderlich)

Empfehlung: vorherige allgemeine Einigung im Organ bzw. Ausschuss über die Sitzungssprache anstreben

Empfehlung: für eventuell erforderliche (Simultan-)Übersetzung mehrere Telefon- oder Sprachleitungen bereitstellen 
Enthält die Satzung oder Geschäftsordnung Besonderheiten hinsichtlich der Durchführung virtueller oder hybrider Sitzungen?

Empfehlung: allgemeine Vorgaben zur form- und fristgerechten Versendung der Einladung samt Tagesordnung und Beschlussvorschlägen sind auch für die Planung virtueller oder hybrider Sitzungen zu beachten, sofern nicht in Satzung oder Geschäftsordnung Ausnahmen bzw. besondere Anforderungen für deren Einberufung stehen

Empfehlung: In Satzung oder Geschäftsordnung Widerspruchsrecht ausschließen (\$108 Abs. 4 AktG)

Sind auch für die virtuellen Sitzungen Präsenzelemente geplant?

Empfehlung: häufig sind Versammlungsleiter, Protokollführer und Vorstandsmitglieder an einem Sitzungsort versammelt, so dass übliche Vorbereitungen für eine Präsenzsitzung (Ort, Anreise, Sitzungsraum, Ausrüstung etc.) in ggf. abgeschwächtem Maß zusätzlich zu beachten sind

Ist für einzelne Tagesordnungspunkte eine Abwesenheit oder zusätzliche Anwesenheit bestimmter Personen erforderlich?

Empfehlung: "virtuelle Lobby“ kann genutzt werden, um das jederzeitige (Wieder-)Eintreten in den virtuellen Sitzungsraum zu ermöglichen

Empfehlung: Personal- oder Aufsichtsratsangelegenheiten können gesammelt zu Beginn oder am Ende der Sitzung behandelt werden

Empfehlung: Gäste bzw. Vortragende zu einzelnen Tagesordnungspunkten (z.B. Compliance Officer, Cybersecurity Officer, Leitung M\&A) erhalten feste Zeitvorgaben oder werden aktiv durch den Protokollführer oder weitere Admin-bzw. IT-Unterstützung über etwaige zeitliche Verschiebungen per Telefon oder E-Mail informiert gehalten

Wird im Ausnahmefall bzw. muss auch die Bilanzsitzung virtuell geplant?

Empfehlung: Auf Teilnahme des Abschlussprüfers an der Bilanzsitzung kann nicht verzichtet werden (\$ 171 Abs. 1 Satz 2 AktG); bei virtueller Teilnahme des Abschlussprüfers daher eine ausführliche Berichterstattung und Möglichkeit der Befragung durch jedes Aufsichtsratsmitglied bzw. eine eingehende Diskussion sicherstellen

Gibt es Vorkehrungen, um die Anwesenheit sicherzustellen und zu überwachen?

Empfehlung: Unterstützung durch Techniker oder sonstiger Spezialisten, die aus technischer Sicht die Videokonferenz begleiten und überwachen erwägenswert

Empfehlung: Aufsichtsratsmitglieder, die Teile der Konferenz aus technischen Gründen verpasst haben, sollten von sich aus auf Informationslücken hinweisen

Empfehlung: Protokollführer sollte im Auge behalten, ob alle als anwesend Verzeichneten ausreichend bzw. möglichst ununterbrochen dem Verlauf der Videokonferenz folgen können

Gibt es Vorkehrungen, um die Abstimmung sicherzustellen und zu überwachen?

Empfehlung: Vor Abstimmungen zusätzlich nochmals darauf achten, dass die als teilnehmend Verzeichneten tatsächlich die Möglichkeit haben, auch an der Abstimmung teilzunehmen

Empfehlung: als Form der Abstimmung „Zuruf" festlegen und nach dem Subtraktionsverfahren vorgehen (nur zur Abgabe von Nein-Stimmen und Enthaltungen auffordern) mit vorheriger transparenter Ankündigung und Erläuterung des Verfahrens und sorgfältiger Kontrolle, ob störungsfreie Teilnahme an der Abstimmung für alle zugeschalteten Mitglieder sichergestellt 


\section{Gibt es Dokumente, die unterzeichnet werden müssen?}

Empfehlung: Bei Bilanzsitzung auch an Unterzeichnung des Jahresabschlusses durch die Vorstandsmitglieder denken (\$ 245 HGB)

Wie sollen Unterlagen im Rahmen der virtuellen Sitzung vorgelegt werden?

Empfehlung: Mangels Möglichkeit ausgedruckter Tischvorlagen entweder rechtzeitige Vorabübermittlung aller Unterlagen oder virtueller Boardroom nutzen

III. Bei Entscheidung zugunsten eines virtuellen Boardrooms zusätzlich zu klären:

Besteht Klarheit über alle für den spezifischen Aufsichtsrat wichtigen Aspekte einer elektronischen Unterlagenführung?

Empfehlung: Mindestaspekte sollten Sicherheit des Boardrooms, Leistung ausreichender und sicherer Zugriffsmöglichkeiten, übersichtliche Struktur der Unterlagendarstellung und -aufbewahrung sein

Empfehlung: zu denken ist auch an eine Archivfunktion, bei der abgelegte Dokumente mit einer aktuellen Sicherheitssoftware verschlüsselt und nur mit dem erforderlichen Berechtigungszertifikat wieder entschlüsselt werden

Empfehlung: zum Schutz des gesamten Datenraums alle Dokumente vor Upload auf Viren überprüfen

Sind die Aufsichtsratsmitglieder ausreichend auf die Verwendung eines virtuellen Boardrooms vorbereitet?

Empfehlung: vorab ausführliche Erläuterungen des elektronischen Boardrooms

Empfehlung: Vorabfrage, ob Aufsichtsratsmitglieder eine Schulung für den elektronischen Boardroom benötigen

Gibt es Unterstützung durch spezialisierte Supportkräfte, an die die Aufsichtsratsmitglieder sich bei technischen und sonstigen Problemen mit dem Boardroom wenden können?

Gibt es ein zentrales Management der Sicherheits- und Authentisierungsregeln (eigenes ITKnowhow vorhanden oder externe Provider)?

Tab. 1: Checkliste für virtuelle Sitzungen des Aufsichtsrats. 